\title{
cGMP elevation: new treatment options for lower urinary tract disorders \\ Peter Sandner
}

\author{
Address: Bayer Schering Pharma, Global Drug Discovery - Common Mechanism Research, Wuppertal, Germany \\ Email: Peter Sandner - peter.sandner@bayerhealthcare.com
}

from 4th International Conference of cGMP Generators, Effectors and Therapeutic Implications

Regensburg, Germany. 19-21 June 2009

Published: II August 2009

BMC Pharmacology 2009, 9(Suppl I):S34 doi:I0.II86/I47I-22I0-9-SI-S34

This abstract is available from: http://www.biomedcentral.com/I47I-22 I0/9/SI/S34

(C) 2009 Sandner; licensee BioMed Central Ltd.

\section{Background}

Although the PDE5 inhibitors were originally targeting cardiovascular disorders, like congestive heart failure or angina, the break-through of this class of drugs was their use in treatment of urological disorders. More than 10 years ago, the clinical introduction of the PDE5i for the treatment of male erectile dysfunction (ED) was a landmark for ED-treatment and is still the gold standard of care. There is accumulating recent evidence that cGMP elevation within the lower urinary tract - outside penile tissue - could effectively regulate prostate and bladder function. This might imply a potential new role of PDE5I for the treatment of benign prostatic syndrome and other lower urinary tract disorders.

\section{Results}

In preclinical experiments it was shown that PDE5 mRNA is expressed in rat bladder and prostate tissue and PDE5 protein is localized in bladder smooth muscle cells. On a functional level, PDE5 inhibition increased cGMP levels in these tissues significantly. Moreover PDE5 inhibitors dose-dependently relaxed rat bladder, prostate and urethra strips. In vivo, PDE5-I dose-dependently reduced non voiding contractions in rats with partial bladder outlet obstruction (BOO) mimicking benign prostatic obstruction in the patient. Non-voiding contractions - a correlate for irritative symptoms - were significantly reduced in BOO-rats after acute and chronic treatment with PDE5 inhibitors. In addition the voiding frequency could be significantly decreased with PDE5-I treatment in anaesthetized and conscious rats with detrusor over-activity.
Finally in conscious dogs voiding frequency was also reduced after PDE5-I treatment. Phase II clinical trials have already confirmed these pre-clinical findings and have shown that in symptomatic BPH patients, sildenafil, tadalafil and vardenafil reduced lower urinary tract symptoms. After chronic daily treatment with these PDE5 inhibitors for 8 to 12 week the IPPS (international prostatic symptom score) was significantly reduced.

\section{Conclusion}

Preclinical and clinical findings show that PDE5 inhibitors could be an effective treatment option for LUTS secondary to $\mathrm{BPH}$ patients in the future. In addition these compounds might have up-side potential for the treatment of overactive bladder. 